\title{
Prospects to observe comets and asteroids using Russian-Cuban Observatory
}

\author{
M. Ibrahimov ${ }^{1}$, D. Bisikalo ${ }^{1}$, A. Fateeva ${ }^{1}$, R. Mata ${ }^{2}$ and O. Pons ${ }^{2}$ \\ 1 Institute of Astronomy, Russian Academy of Sciences, Moscow, Russia \\ 2 Institute of Geophysics and Astronomy, AMA CITMA, Havana, Cuba \\ (E-mail:mansur@inasan.ru)
}

Received: August 10, 2021; Accepted: December 1, 2021

\begin{abstract}
Article reports about prospects to observe comets and asteroids using Russian-Cuban Observatory (RCO). In 2021, RCO wide-field $20 \mathrm{~cm}$ robotic telescope was put into operation. The telescope has $20 \mathrm{~cm}$ aperture, $3.5 \times 3.5$ degree field of view and FLI PL16803 4K CCD camera with a set of $U B V R I$ photometric filters. Telescope itself will be used as a multi-task networking observational tool. Using this Cuban telescope with telescopes located in Russia makes it possible to conduct quasi-day-and-night monitoring of comets and asteroids on an arc of more than 200 degrees.
\end{abstract}

Key words: robotic telescope - astrometry - photometry

\section{Introduction}

Since 2017, the Institute of Astronomy of the Russian Academy of Sciences (INASAN, Moscow, Russia) and the Institute of Geophysics and Astronomy (IGA, Havana, Republic of Cuba) have been implementing a joint international project (Bisikalo et al., 2018). Project main goal is to build the Russian-Cuban Observatory (RCO, see Alonso et al. 2020, Rev. Cubana Fisica, 37, 162). In 2021, the first RCO optical station in Havana was put into operation (Bisikalo et al. 2022, Astronomy Reports, 99, 1, accepted). Main instrument of this station is an optical wide-field $20 \mathrm{~cm}$ robotic telescope. Telescope itself will be used as a multi-task networking observational tool. Brief technical description of $20 \mathrm{~cm}$ telescope and its attachments is provided below. Brief discussion of $20 \mathrm{~cm}$ telescope network using in frame of Russia-Cuba arc is also presented.

\section{IGA optical station and $20 \mathrm{~cm}$ robotic telescope}

A general view of the optical station is shown in Fig. 1. Wide-field $20 \mathrm{~cm}$ robotic telescope with a set of its attachments is shown in Fig. 2. Optical station is located in a single-floor building on the territory of IGA, Havana. Optical station consists of the following main elements: a) automated dome, b) wide-field 


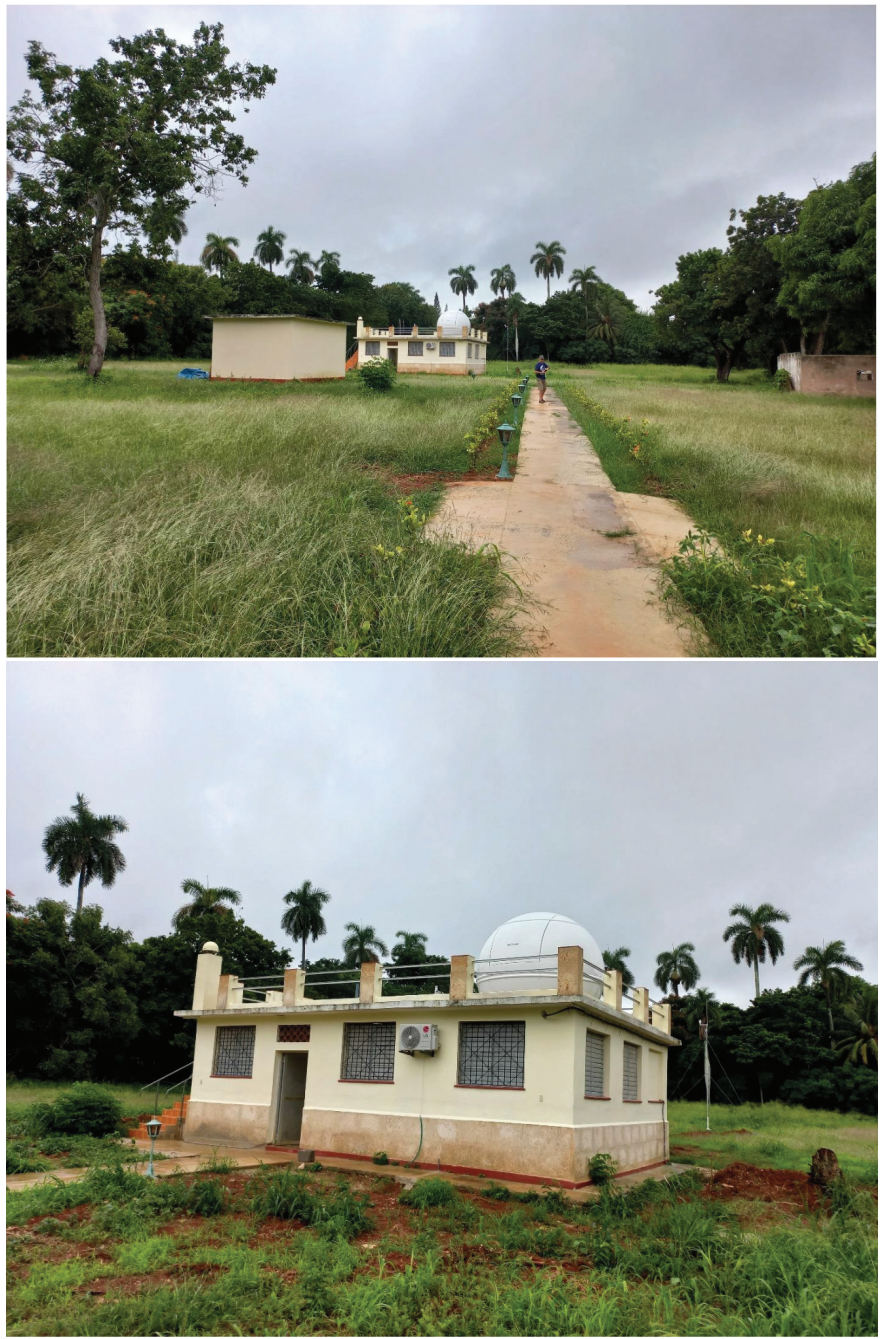

Figure 1. General view of Russian-Cuban Observatory optical station in IGA (Havana, Republic of Cuba).

telescope on an intelligent mount, c) image recording module, d) control, computing, network and meteorological equipment.

An automated 3 -meter dome and a $20 \mathrm{~cm}$ wide-field robotic telescope are installed on the roof of the building Telescope is located in the dome and elevated using 4.2-meter massive concrete column which is separated from the common foundation. The control, computing and network equipment of the observatory is located inside the building. 


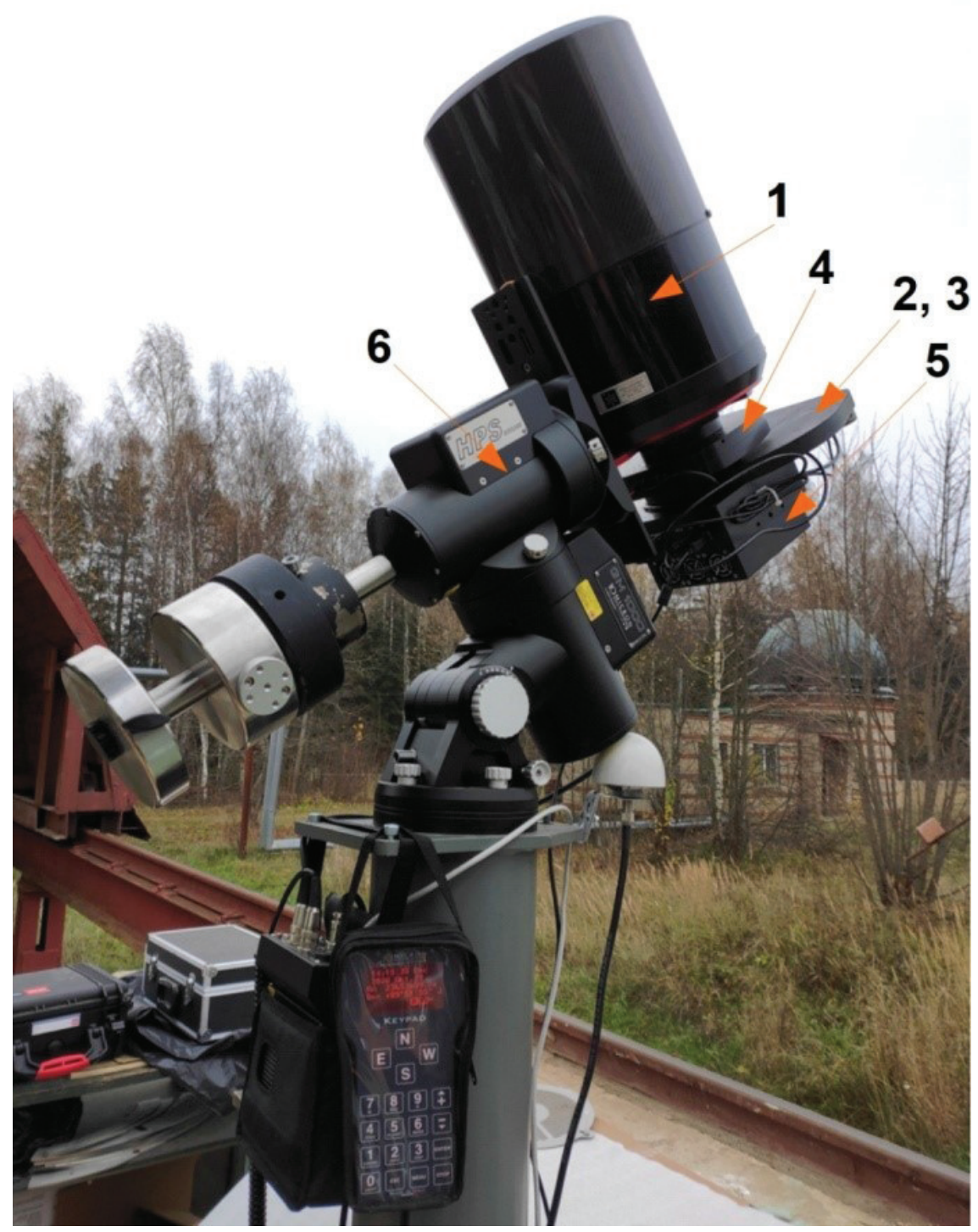

Figure 2. Wide-field $20 \mathrm{~cm}$ robotic telescope with a set of its attachments. Designated: 1 - Officina Stellare Veloce RH200 mirror-and-lens telescope (200 mm aperture, 600 $\mathrm{mm}$ focal length, 3.5x3.5 degree field of view); 2 - 7-position FLI CFW5-7 filter wheel; 3 - set of Johnson-Cousins $U B V R I$ photometric filters $(50 \times 50 \mathrm{~mm}$ size, installed inside FLI CFW5-7 filter wheel); 4 - FLI ATLAS focuser (3", 105000 steps, $85 \mathrm{~nm} / \mathrm{step}, 8.9$ mm stroke); 5 - FLI PL16803 4K CCD camera; 6 - 10 Micron GM1000 HPS mount. 
The following technical equipment was used to robotize observing:

i) 3-meter ScopeDome 3M automated dome;

ii) $20 \mathrm{~cm}$ Officina Stellare Veloce RH200 wide-field telescope with $3.5 \times 3.5$ degree field of view;

iii) 10 Micron GM1000 HPS high-precision equatorial mount with $25 \mathrm{~kg}$ load capacity and absolute position sensors;

iv) FLI PL16803 4K CCD camera with FLI ATLAS automated focusing unit, 7-position FLI CFW5-7 automated filter wheel, and a set of optical filters to realize $U B V R I$ broadband photometric system.

Entire optical station is operated using a software to remote control, observing, data storage and processing. A wide-field optics of $20 \mathrm{~cm}$ robotic telescope were adjusted at INASAN Optical Laboratory. Taking into account some natural limitations due to atmospheric conditions and image scale of 3.08" per pixel for FLI PL16803 4K CCD camera, achieved image quality is FWHM $\approx 5$ " across entire field of view.

\section{20-cm RCO telescope as a multi-task tool: prospects to observe comets and asteroids}

Newly commissioned $20 \mathrm{~cm}$ wide-field robotic telescope is supposed to use as a multi-task astrometric and photometric instrument. A range of solved research tasks is thought to be wide from near-Earth and Solar space tasks (space debris, Solar System small bodies, etc.) to deep-space astrophysical tasks (observations of active and spotted variable stars, detection and/or follow-up observations of Gamma-Ray Burst optical afterglows, etc.). The list of research tasks performed using this telescope includes also observations of various comets and asteroids including Near-Earth Asteroids and Potentially Hazardous Asteroids.

Above described multi-task capability of RCO $20 \mathrm{~cm}$ robotic telescope implies its usage on a permanent base together with the Simeiz Observatory $1 \mathrm{~m}$ Zeiss-1000 and Terskol Observatory 2m Zeiss-2000 telescopes of INASAN Center for collective use (http://www.inasan.ru/divisions/terskol/ckp, (Nikolenko et al., 2019). Also, described network scheme will include $50 \mathrm{~cm}$ telescope of Ussuriysk Department, Institute of Applied Astronomy of the Russian Academy of Sciences and 0.5-1.5m telescopes of two Astronomical Institutes of the Academy of Sciences of Republic of Uzbekistan and Republic of Tajikistan.

The implementation of such a network makes it possible to conduct optical astronomical monitoring using an arc of 214 degree (or 14.3h) in the northern hemisphere of the sky. Taking into account a natural duration of observational night (from 6 to 12 hours for the sites involved), above network will make it possible to conduct quasi- day-and-night monitoring in near-Earth, Solar and deep space. Comets were observed many times in UV by large space telescopes such as Hubble Space Telescope (see a review by Sachkov (2016)). Russian project "Spektr-UF" (The World Space Observatory - Ultraviolet, WSO-UV), 
which is scheduled to launch in 2025, will effectively solve most problems in the field of ultraviolet studies of comets and can become an important research tool (Sachkov et al., 2018, 2019). Data on comets are very important for the study of the formation of planetary systems (Fossati et al., 2014). The wide-field $20 \mathrm{~cm}$ robotic telescope at the Russian-Cuban Observatory may be an instrument for ground support of space observations.

\section{Conclusions}

Paper reports about commissioning of the wide-field $20 \mathrm{~cm}$ robotic telescope at the Russian-Cuban Observatory. Telescope is installed at the optical station in the Institute of Geophysics and Astronomy (Havana, Republic of Cuba). General views of the optical station and its $20 \mathrm{~cm}$ telescope are shown, telescope attachments are described. Multi-task observational projects that will be carried out using RCO $20 \mathrm{~cm}$ telescope are briefly discussed. Russia-Cuba network scheme is described and its prospects to observe Solar System small bodies (comets, asteroids, etc.) are briefly discussed.

Acknowledgements. This work was supported by the Ministry of Science and Higher Education of the Russian Federation, Agreement No. 075-15-2019-1716 of November 20, 2019.

\section{References}

Bisikalo, D. V., Savanov, I. S., Naroenkov, S. A., et al., Perspectives for Distributed Observations of Near-Earth Space Using a Russian-Cuban Observatory. 2018, Astronomy Reports, 62, 367, DOI: 10.1134/S1063772918060021

Fossati, L., Bisikalo, D., Lammer, H., Shustov, B., \& Sachkov, M., Major prospects of exoplanet astronomy with the World Space Observatory-UltraViolet mission. 2014, Astrophysics and Space Science, 354, 9, DOI: 10.1007/s10509-014-2027-3

Nikolenko, I. V., Kryuchkov, S. V., Barabanov, S. I., \& Volkov, I. M., Telescopes of the INASAN Simeiz Observatory: current state and prospects. 2019, INASAN Science Reports, 4, 85, DOI: 10.26087/INASAN.2019.4.2.015

Sachkov, M., Sichevsky, S., Kanev, E., \& Kartashova, A., WSO-UV Field Camera Unit: science case and ground support with 1-m class telescopes. 2019, Contributions of the Astronomical Observatory Skalnate Pleso, 49, 145

Sachkov, M. E., Spectral studies of comets in the ultraviolet range and prospects of the WSO-UV project in these studies. 2016, Solar System Research, 50, 294, DOI: 10.1134/S0038094616040055

Sachkov, M. E., Kartashova, A. P., \& Emel'yanenko, V. V., Prospects for spectral studies of comets: The World Space Observatory - Ultraviolet project. 2018, Planetary Space Science, 164, 75, DOI: 10.1016/j.pss.2018.06.013 